\title{
Controle de tronco e função de membro superior em pacientes com distrofia muscular de Duchenne
}

\author{
Trunk control and upper limb function assessment of the \\ Duchenne muscular dystrophy
}

\author{
Ana Lúcia Yaeko da Silva Santos ${ }^{1}$, Francis Meire Favero ${ }^{2}$, Luis Fernando \\ Grossklauss $^{3}$, Acary Souza Bulle Oliveira ${ }^{3}$, Mariana Cunha Artilheiro ${ }^{4}$, \\ Mariana Callil Voos ${ }^{5}$, Cristina dos Santos Cardoso Sá ${ }^{6}$
}

\footnotetext{
1.Fisioterapeuta, Mestre em Ciências da Saúde Universidade Federal de São Paulo, São Paulo-SP, Brasil. 2. Fisioterapeuta, Professora Afiliada da Universidade Federal de São Paulo, Departamento de Neurologia e Neurocirurgia. São Paulo-SP, Brasil.

3.Neurologista da Universidade Federal de São Paulo. São Paulo-SP, Brasil.

4.Fisioterapeuta, Professora Doutora do Centro Universitário das Faculdades Metropolitanas Unidas. São Paulo-SP, Brasil.

5.Fisioterapeuta, Professora Doutora da Pontifica Universidade Católica. São Paulo-SP, Brasil.

6.Fisioterapeuta, Professora Associada da Universidade Federal de São Paulo. Santos-SP, Brasil.
}

\section{Resumo}

Objetivo. Os objetivos foram avaliar o controle de tronco e o desempenho funcional dos membros superiores (MMSS), e relacionar o nível de controle de tronco, a funcionalidade MMSS e o nível funcional de pacientes com distrofia muscular de Duchenne (DMD). Método. Estudo transversal composto por 35 pacientes diagnosticados com DMD, média de 13,26 anos, submetidos a avaliação do controle de tronco pela Avaliação Segmentar do Controle de Tronco (SATCo), o desempenho de MMSS pelo teste Jebsen Taylor (TJT) e o nível funcional pela escala Vignos. Resultados. O controle de tronco total foi mais prevalente dentre os pacientes, no subteste da escrita do TJT os pacientes tiveram período de tempo prolongado para executá-lo, o nível funcional predominante indicou que os pacientes eram cadeirantes, os quais sentavam eretos e eram capazes de conduzir a cadeira e realizar atividades de vida diária na cama ou na cadeira. Houve correlação entre controle de tronco, funcionalidade de MMSS e nível funcional, e a mesma foi fraca. Conclusão. O estudo caracterizou o controle de tronco e a funcionalidade de MMSS de pacientes com DMD, como também demonstra a relação entre eles e com nível funcional.

Unitermos. Distrofia Muscular de Duchenne; Extremidade Superior; Tronco; Avaliação em Saúde; Fisioterapia

\begin{abstract}
Objective. The aim were trunk control and upper limb function assessment, and the relationship of trunk control, upper limb functional and functional level of the patient with Duchenne muscular dystrophy (DMD). Method. A cross-sectional study of 35 patients diagnosed with DMD, mean age of 13.26 years, submitted to trunk control assessment by the Segmental Assessment of Trunk Control (SATCo), the performance of MMSS by the Jebsen Taylor test (TJT), and the functional level by Vignos scale. Results. Total trunk control was more prevalent among patients, in the TJT writing subtest the patients had an extended period of time to perform it, the predominant functional level indicated that they were wheelchair patients, who sat upright and they were able to lead to chair and perform activities of daily living in bed or in the chair. The correlation between trunk control, upper limb function, and functional level was weak but there was correlation. Conclusion. The study characterizes the trunk control and upper limb functional of patients with DMD, but also demonstrates a relationship between them and the functional level.
\end{abstract}


Keywords. Duchenne Muscular Dystrophy; Upper Extremity; Torso; Health Evaluation; Physical Therapy Specialty.

Trabalho realizado na Universidade Federal de São Paulo, UNIFESP, Departamento de Neurologia/Neurocirurgia, São Paulo-SP, Brasil.

Conflito de interesse: não Recebido em: 2018

Aceito em: 2018

Endereço para correspondência: Cristina S C de Sá. Departamento de Ciências do Movimento Humano, Universidade Federal de São Paulo, Av. Ana Costa, 95, Vila Mathias, Santos-SP, CEP 11050-240, Brasil. Email: cristina.sa@uol.com.br

\section{INTRODUÇÃO}

A distrofia muscular de Duchenne (DMD) causa fraqueza muscular progressiva, que prejudica a independência funcional $e$, consequentemente, a qualidade de vida desses pacientes no domínio físico, emocional e social ${ }^{1}$.

A independência funcional relaciona-se diretamente com o sucesso no desempenho das tarefas de autocuidado, transferências, locomoção, trabalhar e/ou estudar. Conforme a progressão da fraqueza muscular de proximal para distal $^{2}$, os pacientes apresentam-se cada vez mais dependentes de cuidados para as atividades de autocuidado e locomoção ${ }^{3}$. Tais tarefas envolvem controle de tronco e a função adequada dos membros superiores (MMSS).

O controle de tronco está atrelado à função dos MMSS, desde o desenvolvimento motor do lactente. À medida que o lactente consegue controlar a cabeça e o tronco em determinada postura, seja no decúbito ventral, sedestação ou ortostatismo, ele ganha o alcance funcional do membro 
superior (MS). Estudos confirmam que o suporte do tronco influencia a qualidade do alcance funcional em lactentes ${ }^{4,5}$.

Dessa forma, o controle de tronco e a função de MMSS retratam a funcionalidade dos pacientes, porém há escassez na literatura de estudos que avaliem de forma conjunta tais habilidades em pacientes com DMD. Esse estudo avaliou o controle de tronco e a função de MS na DMD, com o intuito de permitir ao fisioterapeuta melhor compreensão dessa relação e planejamento de tratamento apropriado e específico para o déficit motor apresentado, visando melhor desempenho funcional, maior participação social e qualidade de vida.

Este estudo verificou o controle de tronco e o desempenho funcional dos MMSS, e relacionou o nível de controle de tronco, a funcionalidade de MMSS e o estadiamento da doença de paciente com DMD.

\section{MÉTODO}

\section{Participantes}

Trata-se de estudo transversal composto por pacientes, do sexo masculino, avaliados no Ambulatório de Investigações nas Doenças Neuromusculares do Departamento de Neurologia e Neurocirurgia da Universidade Federal de São Paulo (UNIFESP), na região sudeste do Brasil. Os pacientes incluídos no estudo foram diagnosticados com DMD por biópsia muscular ou teste de ácido desoxirribonucleico (DNA). Entretanto, foram 
excluídos pacientes submetidos a procedimentos cirúrgicos prévios nos MMSS e/ou na coluna vertebral, com dificuldade para compreensão de comandos verbais simples, ou pacientes com déficits cognitivos $(M E E M<10$ pontos) ${ }^{6}$, visuais e/ou auditivos que impossibilitem a aplicação do protocolo de pesquisa, ou aqueles que por qualquer motivo interromperam a avaliação. Este estudo foi aprovado pelo Comitê de Ética em Pesquisa da UNIFESP, sob o parecer 1.367.911.

\section{Procedimentos}

O aspecto cognitivo foi avaliado por meio da aplicação do Mini Exame do Estado Mental (MEEM), teste validado para a população brasileira ${ }^{7,8}$. Pacientes com pontuação menor que 10 pontos foram excluídos do estudo ${ }^{6}$.

O nível do controle de tronco foi estabelecido pela Avaliação Segmentar do Controle de Tronco (SATCo-Br) ${ }^{9}$, a qual varia de 1 a 7 , sendo nível 1 correspondente ao controle de cabeça, nível 2 ao torácico superior, nível 3 ao torácico médio, nível 4 ao torácico inferior, nível 5 ao lombar alto, nível 6 ao lombar inferior e 7 ao nível de controle total de tronco. Para definir o nível de controle de tronco de cada paciente, foi considerado o último nível em que todos os três componentes (estático, ativo e reativo) estivessem presentes ${ }^{10}$. Para sua aplicação, foi utilizado banco de altura apropriada do paciente e um sistema de cintas ligado a pelve, para assegurar que a pelve permaneça em posição neutra. 
A avaliação da função de MS foi realizada pelo teste Jebsen Taylor (TJT), o qual é composto de sete subtestes ${ }^{11}$ : subteste 1, escrita (1T1 e 1T2); subteste 2, virar cartão ( $2 \mathrm{~T} 1$ e $2 \mathrm{~T} 2$ ); subteste 3 , pegar objetos pequenos comuns (3T1 e 3T2); subteste 4, simular alimentação (4T1 e 4T2); subteste 5, pegar peças de jogo de dama (5T1 e 5T2); subteste 6 pegar objetos largos e leves (6T1 e 6T2); subteste 7 , pegar objetos largos e com peso. Porém 0 subteste 7 foi retirado a fim de tornar o TJT aplicável às distrofias musculares ${ }^{10}$. Todos os subtestes foram realizados na sequência estabelecida para os dois MMSS, iniciando pelo MS não dominante (T1) e na sequência pelo MS dominante (T2). Nas tentativas em que algum voluntário não conseguiu completar algum dos subtestes, 0 mesmo foi excluído e a cronometragem não analisada. Cada tentativa foi filmada e mensurada quantitativamente por meio de cronômetro.

Escala Vignos foi utilizada para classificar o estadiamento da doença dos pacientes. A pontuação da escala varia de 0 a 10 , quanto maior a pontuação pior 0 desempenho funcional do paciente ${ }^{13}$.

\section{Análise Estatística}

Foi realizada análise descritiva, distribuição de frequência, para as variáveis: nível de controle de tronco, nível funcional (estadiamentos da doença); e média e desvio padrão para os subtestes do TJT. O teste Kolmogorov-Smirnov verificou a normalidade dos dados. 
Posteriormente, foi realizado 0 teste de Pearson. A correlação foi testada entre o nível funcional e nível de controle de tronco, assim o tempo de execução de cada subteste da TJT. Também entre o nível de controle de tronco e tempo de execução de cada subteste da TJT. As correlações foram classificadas como muito forte $(r=0,9)$, forte $(0,7 \leq r<0,9)$, moderado $(0,5 \leq r<0,7)$, fraca $(0,3 \leq r<0,5)$ e muito fraca $(0 \leq r<0,3)^{14}$; valores $p \leq 0,05$ foram considerados estatisticamente significativos. Para as análises foi utilizado o software SPSS v.19.

\section{RESULTADOS}

Foram avaliados 45 pacientes, contudo, foram incluídos 35 pacientes. Sendo excluídos 10 pacientes, pois sete não sabiam escrever, e outros três, devido a déficit cognitivo que impossibilitou a compreensão de comandos verbais simples.

Os pacientes com DMD apresentaram idade média de

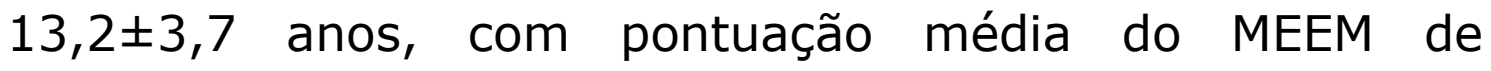
$24,2 \pm 5,8$ pontos. A maioria dos pacientes apresentou Vignos $7(48,6 \%)$, indicando que o paciente era cadeirante, sentava ereto, podia conduzir a cadeira e era capaz de realizar atividades de vida diária na cama ou na cadeira $A$ escoliose estava presente em $66 \%$ dos pacientes (Tabela 1). 
Tabela 1. Distribuição de frequência absoluta e relativa da variável escoliose e Vignos (estadiamentos da doença).

\begin{tabular}{llc}
\hline \multirow{2}{*}{ Escoliose } & \multicolumn{2}{c}{ Pacientes (n=35) } \\
\hline & Presença & $23(66,0 \%)$ \\
\cline { 2 - 3 } Ausência & $12(34,3 \%)$ \\
\hline \multirow{2}{*}{ Escala Vignos 1} & $1(3,0 \%)$ \\
\cline { 2 - 3 } & Vignos 2 & $3(8,6 \%)$ \\
\cline { 2 - 3 } & Vignos 3 & $2(5,7 \%)$ \\
\cline { 2 - 3 } & Vignos 5 & $3(8,6 \%)$ \\
\cline { 2 - 3 } & Vignos 7 & $7(23,0 \%)$ \\
\hline
\end{tabular}

Vignos 1, paciente sobe escadas sem auxílio do corrimão; Vignos 2, paciente sobe escadas com auxílio do corrimão; Vignos 3, paciente sobe 8 degraus por mais que 25 segundos; Vignos 4, paciente não sobe escadas; Vignos 5, paciente anda sem auxílio mas não sobe escadas ou levanta da cadeira; Vignos 6, paciente anda apenas com auxílio externo; Vignos 7 , paciente na cadeira de rodas, consegue tocar a sua cadeira e realizar as atividades de vida diária; Vignos 8, paciente na cadeira de rodas e não consegue realizar as atividades de vida diária sem auxílio.

Nota-se que $40 \%$ dos pacientes apresentou nível 1 de controle, indicando que possuíam apenas controle de cabeça, já 34,3\% nível 7 de controle de tronco, indicando controle de tronco completo, ou seja, com os três componentes do controle: estático, dinâmico e reativo; em todos os níveis. Contudo $11,4 \%$ dos pacientes apresentou nível 4 de controle, indicando controle torácico inferior, ou controle de tronco superior (Tabela 2). 
Tabela 2. Distribuição de frequência absoluta e relativa da variável controle de tronco.

\begin{tabular}{ll}
\hline SATCo-BR & Pacientes $(\mathbf{n}=\mathbf{3 5})$ \\
\hline Nível 1: controle de cabeça & $14(40,0 \%)$ \\
\hline Nível 2: controle torácico superior & $2(5,7 \%)$ \\
\hline Nível 3: controle torácico médio & $1(2,9 \%)$ \\
\hline Nível 4: controle torácico inferior & $4(11,4 \%)$ \\
\hline Nível 5: controle lombar superior & $2(5,7 \%)$ \\
\hline Nível 7: controle de tronco total & $12(34,3 \%)$ \\
\hline
\end{tabular}

SATCo-BR: Avaliação Segmentar do Controle de Tronco.

A Tabela 3 demonstra que dentre todos os subtestes, a condição T2 revelou-se mais rápida que a condição T1. No subteste de escrita tanto com o MS dominante como o não dominante os pacientes demoraram mais tempo para executar. Entretanto, o subteste de pegar peças de dama foi o mais rápido para executar independe do MS.

A Tabela 4 descreve a correlação entre o controle de tronco, o estadiamento da doença, pela escala Vignos, e tempo de execução dos subtestes do TJT. De maneira geral, a correlação foi muito fraca entre o estadiamento da doença e os subtestes do TJT, e fraca entre o controle de tronco e os subtestes do TJT com o MS dominante. Porém a correlação foi moderada entre o controle de tronco e o estadiamento da doença, bem como o controle de tronco e o subteste de pegar objetos largos e leves com o MS não dominante. 
Tabela 3. Média, Desvio padrão e valores máximos e mínimos dos tempos (em segundos - s) dos subtestes do TJT .

\begin{tabular}{lll}
\hline Subtestes & Média \pm DP & Mín-Máx \\
\hline 1T1(s) & $93,6 \pm 58,8$ & $38-274$ \\
\hline 1T2(s) & $63,9 \pm 58,3$ & $24-246$ \\
\hline 2T1(s) & $16,7 \pm 11,6$ & $3,4-66,2$ \\
\hline 2T2(s) & $13,8 \pm 9,91$ & $3,6-43,3$ \\
\hline 3T1(s) & $21,8 \pm 24,6$ & $5,6-122,6$ \\
\hline 3T2(s) & $16,9 \pm 14,2$ & $5,1-68,7$ \\
\hline 4T1(s) & $41,2 \pm 54,5$ & $5,0-234,6$ \\
\hline 4T2(s) & $27,1 \pm 35,9$ & $6,0-133,6$ \\
\hline 5T1(s) & $13,1 \pm 8,1$ & $2,6-40,2$ \\
\hline 5T2(s) & $10,5 \pm 5,9$ & $2,3-34,4$ \\
\hline 6T1(s) & $18,3 \pm 15,9$ & $4,7-69,9$ \\
\hline 6T2(s) & $12,5 \pm 8,5$ & $4,2-37,3$
\end{tabular}

DP: desvio padrão; s:segundos; Máx: valor máximo; Mín: valor mínimo; 1T1: escrita com membro superior não dominante; 1T2: escrita com membro superior dominante; $2 \mathrm{~T} 1$ : virar cartões com membro superior não dominante; 2T2: virar cartões com membro superior dominante; 3T1: pegar objetos pequenos e comuns com membro superior não dominante; 3T2: pegar objetos pequenos e comuns com membro superior dominante; 4T1: simular a alimentação com membro superior não dominante; 4T2: simular a alimentação com membro superior dominante; 5T1: pegar peças de jogo de dama com membro superior não dominante; 5T2: pegar peças de jogo de dama com membro superior não dominante; 6T1: pegar objetos largos e leves com membro superior não dominante; 6T2: pegar objetos largos e leves com membro superior dominante. 
Tabela 4: Correlação entre a escala Vignos, SATCo e subtestes doTJT.

\begin{tabular}{|c|c|c|}
\hline Correlação & Valor de $r$ & Valor de $p$ \\
\hline Vignos x SATCo & $-0,557$ & $0,001^{*}$ \\
\hline Vignos X 1T1 & 0,001 & 0,996 \\
\hline$\overline{\text { SATCO X 1T1 }}$ & $-0,020$ & 0,908 \\
\hline Vignos X 1T2 & 0,290 & 0,828 \\
\hline$\overline{\text { SATCO X 1T2 }}$ & $-0,288$ & 0,093 \\
\hline Vignos x 2T1 & 0,296 & 0,084 \\
\hline$\overline{\text { SATCO X 2T1 }}$ & $-0,258$ & 0,135 \\
\hline Vignos $\times 2 \mathrm{~T} 2$ & 0,171 & 0,326 \\
\hline$\overline{\text { SATCO X 2T2 }}$ & $-0,364$ & $0,031^{*}$ \\
\hline Vignos x 3T1 & 0,250 & 0,147 \\
\hline$\overline{\text { SATCO X 3T1 }}$ & $-0,199$ & 0,251 \\
\hline Vignos x 3T2 & 0,305 & 0,075 \\
\hline$\overline{\text { SATCO X } 3 T 2}$ & $-0,318$ & 0,062 \\
\hline Vignos x 4T1 & 0,305 & 0,074 \\
\hline$\overline{\text { SATCO X 4T1 }}$ & $-0,378$ & $0,025^{*}$ \\
\hline Vignos x 4T2 & 0,282 & 0,100 \\
\hline$\overline{\text { SATCO X 4T2 }}$ & $-0,394$ & $0,019 *$ \\
\hline Vignos X 5T1 & 0,148 & 0,395 \\
\hline$\overline{\text { SATCO X 5T1 }}$ & $-0,025$ & 0,888 \\
\hline Vignos X 5T2 & 0,350 & $0,039 *$ \\
\hline$\overline{\text { SATCO X 5T2 }}$ & $-0,236$ & 0,172 \\
\hline Vignos x 6T1 & 0,395 & $0,019 *$ \\
\hline$\overline{\text { SATCO X 6T1 }}$ & $-0,632$ & $0,001^{*}$ \\
\hline Vignos x 6T2 & 0,177 & $0,309 *$ \\
\hline$\overline{\text { SATCO X 6T2 }}$ & $-0,317$ & 0,064 \\
\hline
\end{tabular}

*: valores de $p \leq 0,05$ foram considerados estatisticamente significativos. 1T1: escrita com membro superior não dominante; 1T2: escrita com membro superior dominante; $2 \mathrm{~T} 1$ : virar cartões com membro superior não dominante; 2T2: virar cartões com membro superior dominante; 3T1: pegar objetos pequenos e comuns com membro superior não dominante; 3T2: pegar objetos pequenos e comuns com membro superior dominante; 4T1: simular a alimentação com membro superior não dominante; 4T2: simular a alimentação com membro superior dominante; 5T1: pegar peças de jogo de dama com membro superior não dominante; 5T2: pegar peças de jogo de dama com membro superior não dominante; 6T1: pegar objetos largos e leves com membro superior não dominante; 6T2: pegar objetos largos e leves com membro superior dominante. $r=0,9$ : muito forte; $0,7 \leq r<0,9$ : forte; $0,5 \leq r<0,7$ : moderado; $0,3 \leq r<0,5$ : fraca; $0 \leq r<0,3$ : muito fraca. 


\section{DISCUSSÃO}

Nosso estudo verificou $\mathrm{O}$ controle de tronco e funcionalidade de MMSS. A escala SATCo utilizada para avaliar o nível de controle de tronco possibilitou verificar as diferenças do controle de tronco entre os pacientes com DMD. A avaliação do controle de tronco indicou que a maioria dos pacientes encontravam-se nos extremos da escala SATCo, i.e.; controle de cabeça e controle de tronco total; e a minoria variou entre controle torácico superior a controle lombar superior. A confiabilidade da escala foi confirmada pelo estudo de Sá et al. ${ }^{15}$, no qual a escala mostrou-se eficaz para detectar as diferenças do nível de controle de tronco de pacientes com DMD. Logo, essa avaliação pode ser utilizada para identificar o nível de controle de tronco dos pacientes com DMD, e permite auxiliar no estabelecimento de condutas mais específicas para esses pacientes.

O TJT verificou o desempenho funcional dos MMSS e apontou que houve mais facilidade dos pacientes para realizar os subtestes com o MS dominante e o subteste de pegar peças de dama. Já o subteste de escrita e a simulação da alimentação foram mais difíceis para os pacientes. Tais dados corroboram com o estudo de Artilheiro et al. ${ }^{16}$, que também identificou a facilidade de execução dos subtestes com o MS dominante, porém não houve diferença, segundo os autores, entre utilização do MS dominante e o não dominante, exceto no subteste de 
escrita que foi significativamente mais rápido com o MS dominante, o que era esperado.

O subteste de escrita e simulação da alimentação exigem adequada estabilidade axial e proximal de MS; amplitude de movimento de ombro, cotovelo, punho e dedos; e coordenação no recrutamento muscular de agonistas e antagonistas da mão. Tais características estão comprometidas pela fraqueza muscular e deformidades articulares dos pacientes com $\mathrm{DMD}^{17}$, as quais influenciaram no tempo e na precisão de execução dos subtestes. Foi identificada forte correlação entre o MS dominante e o não dominante em cada subteste do $\mathrm{TJT}^{16}$. Isto indica que pesquisadores e clínicos podem avaliar a funcionalidade dos MMSS dos pacientes com DMD pelo TJT, solicitando somente a execução dos subtestes com o MS dominante.

Este estudo relacionou o controle de tronco e a funcionalidade de MMSS de pacientes com DMD. O alinhamento ativo do tronco corresponde ao controle de tronco, o qual envolve esforço muscular necessário para manter o tronco alinhado contra a força da gravidade e perturbações internas ou externas para a execução de determinada tarefa, ou seja, ocorre ativação dos músculos estabilizadores do tronco antes do movimento de flexão, extensão e abdução de $\mathrm{MMSS}^{18}$. Dessa forma, a hipótese era que haveria correlação entre o controle de tronco e a função de MMSS, porém houve correlação fraca entre a 
SATCo e os subtestes do TJT, exceto para o subteste de pegar objetos largos e leves com MS não dominante com correlação moderada entre as escalas, ou seja, quanto melhor o controle de tronco mais rápida a execução deste subteste. Há escassez na literatura de estudos que correlacionem o controle de tronco e a função de MMSS em distrofinopatias. Os achados desse estudo permitem afirmar que quanto melhor o controle de tronco, melhor 0 desempenho no subteste de pegar objetos largos e leves com MS não dominante. Tal informação corrobora para a estimulação do controle de tronco e a função de MMSS no planejamento terapêutico. Portanto o controle de tronco e a função de MMSS são essenciais para a independência funcional $^{19}$, visto que os pacientes com DMD progressivamente serão dependentes de seus cuidadores ${ }^{20}$. Os demais subtestes puderam não apresentar maiores correlações com o controle de tronco pela diferença da forma de avaliação das escalas. O TJT mensura a função de MMSS de forma qualitativa, e a SATCo avalia o controle de tronco de maneira qualitativa.

A progressão da fraqueza muscular na DMD é de proximal para distal, com prejuízo na musculatura antigravitária de membros inferiores (MMII) e tronco. Em consequência por volta dos 13 anos de idade ocorre à perda da habilidade da deambulação desses pacientes ${ }^{21}$. Esse estudo contribuiu com este achado da literatura, pois a nossa amostra teve em média 13,2 anos de idade e em sua maioria com pontuação 7 na escala Vignos, indicando que 
os pacientes eram cadeirantes, sentavam ereto, eram capazes de conduzir a cadeira e realizar atividades de vida diária na cama ou na cadeira de forma independente. Apesar da nossa amostra apresentar fraqueza de MMII (Vignos 7) e de tronco (controle de cabeça), todos foram capazes de completar todos os subtestes do TJT com o MS dominante e o MS não dominante. Entretanto, o TJT não possibilita a avaliação da qualidade do movimento desses pacientes para completar os subtestes.

O uso as cadeira de rodas permite que esses pacientes possam se locomover dentro da casa, da escola, do trabalho, do ambulatório médico, bem como deslocar-se entre esses locais e áreas de lazer, com independência. Apesar dos benéficos, os longos períodos desses pacientes na cadeira de rodas sem a atenção e o cuidado de um profissional da saúde habilitado associado com a história de progressão natural da doença, pode levar a encurtamentos da musculatura flexora de MMII, escoliose, disfunção do sistema gastrointestinal, respiratório, cardíaco ${ }^{21}$. A escoliose tende a aparecer em 1 a 2 anos após o paciente ter perdido a marcha, sendo que quando mais lenta a progressão da curvatura maior a expectativa de vida dos pacientes ${ }^{22}$, devido ao fato de a progressão da curvatura escoliótica prejudicar significativamente a função respiratória ${ }^{23}$. A presença da escoliose e a locomoção independente por cadeira de rodas foram características da nossa amostra. 
Baptista et al. ${ }^{24}$ relatam que pacientes com DMD têm déficit nos ajustes antecipatórios e compensatórios, pois o sistema musculoesquelético não gera forças de estabilização para antecipar ou recuperar o equilíbrio. Esse estudo, encontramos que $66 \%$ dos pacientes avaliados apresentavam escolioses e observamos a dificuldade dos pacientes em manter o controle de tronco associado com a utilização deste para cumprir as tarefas de MMSS. Acreditamos que o aparecimento da escoliose esteja atrelado ao auxílio do movimento de tronco para executar as tarefas funcionais do cotidiano dos pacientes com DMD.

Houve quantidade considerável de pacientes que apresentou somente controle de cabeça e escore Vignos 8, que caracteriza-se por pacientes cadeirantes que necessitam de assistência para atividades com o MS em sedestação ou decúbito dorsal. Os pacientes com Vignos 8 realizam o subteste 3 e 4 arrastando os objetos até o recipiente que deveriam ser colocados, essa adaptação também foi observada pelo estudo de Artilheiro et al. ${ }^{12}$. Os pacientes com Vignos menor ou igual 7, apresentaram bom controle de tronco (controle de tronco total e controle lombar superior) e eram capazes de realizar os subtestes do TJT e suas atividades de vida diária. Embora essa correlação foi fraca com os subtestes do TJT e a escala Vignos, diferente do que esperávamos. No entanto a SATCo e Vignos tiveram correlação moderada, ou seja, quanto pior o controle de tronco, pior o estadiamento da doença. $O$ estudo de Sá et al. ${ }^{15}$ demonstrou que os pacientes com o 
mesmo estadiamento da doença tinham níveis de controle de tronco diferentes.

Nosso estudo limitou-se quanto ao número de pacientes avaliados, o que dificultou diferenciar os pacientes em grupos, como deambuladores e não deambuladores. Essa divisão poderia esclarecer os pacientes nas extremidades da SATCo e sua correlação com o desempenho dos MMSS. Entretanto esse é o primeiro estudo a relacionar o controle de tronco e a função com o MMSS, como também expressa a importância dos profissionais de saúde atentarem-se para o controle de tronco desses pacientes, pois dessa maneira irão estabelecer condutas que controle a progressão do estadiamento da doença, a redução das escolioses e os seus prejuízos na expectativa dos pacientes com DMD.

\section{CONCLUSÃO}

Conclui-se que no subteste da escrita e alimentação, os pacientes tiveram período de tempo prolongado para executá-los, já o subteste de pegar peças de dama eles apresentaram mais facilidade. Houve pacientes tanto com controle de cabeça, como controle completo de tronco, de acordo com o estadiamento da doença, ou seja, quanto mais avançada à doença pior o controle de tronco. Não foi possível correlacionar o controle de tronco e o desempenho dos MMSS. Sugerimos novos estudos que abordem a 
correlação do controle de tronco e a função dos MMSS, com um teste de MS qualitativo.

\section{REREFÊNCIAS}

1.Bendixen RM, Senesac C, Lott DJ, Vandenborne K. Participation and quality of life in children with Duchenne muscular dystrophy using the International Classification of Functioning, Disability, and Health. Health Qual Life Outcomes 2012;10:43.

http://dx.doi.org/10.1186/1477-7525-10-43.

2.Emery AE. The muscular dystrophies. Lancet 2002;359:687-95. http://dx.doi.org/10.1016/S0140-6736(02)07815-7

3.Santos NM, Rezende M, Terni A, Hayashi MCB, Fávero FM, Quadros $A A J$, et al. Perfil clínico e funcional dos pacientes com distrofia muscular de Duchenne assistidos na Associação Brasileira de Distrofia Muscular (ABDIM). Rev Neurocienc 2006;14:15-22.

4.Rachwani J, Santamaria V, Saavedra SL, Wood S, Porter F, Woollacott $\mathrm{MH}$. Segmental trunk control acquisition and reaching in typically developing infants. Exp Brain Res 2013;228:131-9. http://dx.doi.org/10.1007/s00221-013-3544-y

5.Rachwani J, Santamaria V, Saavedra SL, Woollacott MH. The development of trunk control and its relation to reaching in infancy: a longitudinal study. Front Hum Neurosci 2015;9:94. http://dx.doi.org/10.3389/fnhum.2015.00094

6.Voos M, Favero F, Dias K, Artilheiro M, Oliveira A, Caromano F. Dissociation between motor and cognitive skills in subjects with Duchenne muscular dystrophy. Neuromuscul Disord 2015;25(suppl 2):S306.

7.Anthony JC, LeResche L, Niaz U, von Korff MR, Folstein MF. Limits of the 'Mini-Mental State' as a screening test for dementia and delirium among hospital patients. Psychol Med 1982;12:397-408. http://dx.doi.org/10.1017/s0033291700046730

8.Brucki SM, Nitrini R, Caramelli P, Bertolucci PH, Okamoto IH. Sugestões para o uso do mini-exame do estado mental no Brasil. Arq Neuropsiquiatr 2003;61:777-81. http://dx.doi.org/10.1590/s0004282x2003000500014

9.Sá CdSCd, Fávero FM, Voos MC, Choren F, Carvalho RdP. Brazilian version of the Segmental Assessment of Trunk Control (SATCo). Fisioter Pesqui 2017;24:89-99. http://dx.doi.org/10.1590/18092950/16955824012017

10.Butler PB, Saavedra S, Sofranac M, Jarvis SE, Woollacott MH. Refinement, reliability, and validity of the segmental assessment of trunk control. Pediatr Phys Ther 2010;22:246-57. http://dx.doi.org/10.1097/PEP.0b013e3181e69490 
11.Jebsen RH, Taylor N, Trieschmann RB, Trotter MJ, Howard LA. An objective and standardized test of hand function. Arch Phys Med Rehabil 1969;50:311-9.

12. Artilheiro MC, Favero FM, Caromano FA, Oliveira ASB, Carvas NJ, Voos MC, et al. Reliability, validity and description of timed performance of the Jebsen-Taylor Test in patients with muscular dystrophies. Braz J Phys Ther 2018;22:190-7. http://dx.doi.org/10.1016/j.bjpt.2017.09.010

13.Vignos PJ, Jr., Archibald KC. Maintenance of ambulation in childhood muscular dystrophy. J Chronic Dis 1960;12:273-90. http://dx.doi.org/10.1016/0021-9681(60)90105-3

14. Hulle SB, Cummings SR, Browner WS, Grady DG, Newman TB. Delineando a pesquisa clinica: uma abordagem epidemiológica. 3a ed. São Paulo: Artmed; 2008.

15.Sá CSC, Fagundes IK, Araujo TB, Oliveira AS, Favero FM. The relevance of trunk evaluation in Duchenne muscular dystrophy: the segmental assessment of trunk control. Arq Neuropsiquiatr 2016;74:791-5. http://dx.doi.org/10.1590/0004-282X20160124

16. Artilheiro MC, Sa CSC, Favero FM, Caromano FA, Voos MC. Patients with Duchenne and Becker muscular dystrophies are not more asymmetrical than healthy controls on timed performance of upper limb tasks. Braz J Med Biol Res 2017;50:e6031. http://dx.doi.org/10.1590/1414-431X20176031

17. Bartels B, Pangalila RF, Bergen MP, Cobben NA, Stam HJ, Roebroeck ME. Upper limb function in adults with Duchenne muscular dystrophy. J Rehabil Med 2011;43:770-5.

http://dx.doi.org/10.2340/16501977-0841

18. Hodges $P$, Cresswell A, Thorstensson A. Preparatory trunk motion accompanies rapid upper limb movement. Exp Brain Res 1999;124:69-79. http://dx.doi.org/10.1007/s002210050601

19.Shumway-Cook A, Woollacott M. Alcance, preensão e manipulação normais. Controle Motor: teoria e aplicações práticas. $3^{a}$ ed. Chaim MCB. Barueri: Manole, 2010, p.449-73.

20.Landfeldt E, Lindgren P, Bell CF, Guglieri M, Straub V, Lochmuller $\mathrm{H}$, et al. Quantifying the burden of caregiving in Duchenne muscular dystrophy. J Neurol 2016;263:906-15. http://dx.doi.org/10.1007/s00415-016-8080-9

21.Bushby K, Finkel R, Birnkrant DJ, Case LE, Clemens PR, Cripe L, et al. Diagnosis and management of Duchenne muscular dystrophy, part 1: diagnosis, and pharmacological and psychosocial management. Lancet Neurol 2010;9:77-93. http://dx.doi.org/10.1016/S14744422(09)70271-6

22.Smith AD, Koreska J, Moseley CF. Progression of scoliosis in Duchenne muscular dystrophy. J Bone Joint Surg Am 1989;71:106674.

23.Yamashita T, Kanaya K, Yokogushi K, Ishikawa $Y$, Minami R. Correlation between progression of spinal deformity and pulmonary function in Duchenne muscular dystrophy. J Pediatr Orthop 
2001;21:113-6. $\quad$ http://dx.doi.org/10.1097/00004694-20010100000022

24.Baptista CR, Costa AA, Pizzato TM, Souza FB, Mattiello-Sverzut AC. Postural alignment in children with Duchenne muscular dystrophy and its relationship with balance. Braz J Phys Ther 2014;18:119-26. http://dx.doi.org/10.1590/S1413-35552012005000152 